\title{
Efecto de los factores de innovación en el desempeño económico de los talleres artesanales de la zona metropolitana de Zacatecas
}

\author{
The effect of the factors of innovation in the economic performance \\ of the craft workshops of the metropolitan area of Zacatecas
}

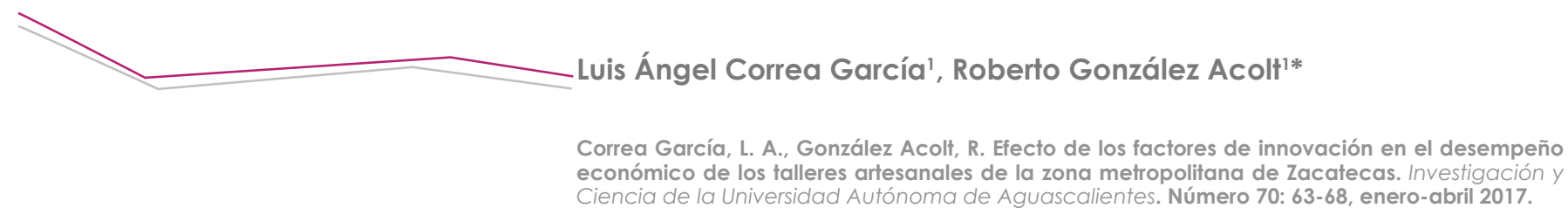

\section{RESUMEN}

El propósito de este trabajo fue analizar el efecto de los factores de innovación sobre el desempeño económico de los talleres artesanales de la zona metropolitana de Zacatecas, para ello se aplicó un cuestionario a 124 artesanos, posteriormente se realizó un análisis de regresión múltiple y se emplearon pruebas de restricciones de exclusión para examinar si un grupo de variables -ligadas a diferentes tipos de innovación- influyeron en los ingresos que perciben los trabajadores de esta zona. Los resultados mostraron que la variable innovación manifiesta efecto en el producto, la organización y mercadotecnia con los ingresos mensuales de estos operarios.

\section{ABSTRACT}

The purpose of this paper is to analyze the effect of the factors of innovation on the economic performance, the craft workshops of the metropolitan area of Zacatecas, for this a questionnaire was administered to 124 artisans, a multiple regression analysis was subsequently performed. A restriction of exclusion tests was used to examine a group of variables linked to different types of innovation- influencing

Palabras clave: innovación, desempeño económico, taller artesanal, zona metropolitana de Zacatecas.

Keywords: innovation, economic performance, craft workshop, handcrafts, metropolitan area of Zacatecas.

Recibido: 28 de enero de 2016, aceptado: 16 de diciembre de 2016

Departamento de Economía, Centro de Ciencias Económicas y Administrativas, Universidad Autónoma de Aguascalientes, México.

* Autor para correspondencia: rgonza@correo.vaa.mx revenues that the artisans in this area perceived. The results showed that variable innovation states effect on the product, organization and marketing with monthly incomes of these artisans.

INTRODUCCIÓN

El estudio en los talleres artesanales constituye un importante desafío, debido a que en la literatura son considerados empresas de subsistencia (Toledo López et al., 2012). En la zona metropolitana de Zacatecas las microempresas artesanales son una de las mayores riquezas de la entidad, a pesar de que su continuidad está en peligro de desaparecer, ya que las nuevas generaciones no se interesan en continuar con la tradición artesanal (Burciaga, 2010). A través de los años, el trabajo de los artesanos se ha desarrollado en medio de bastantes dificultades que frenan su competitividad, debido a que disponen de menos recursos materiales, lo que provoca que estos negocios tengan que realizar innovaciones en sus prácticas productivas.

Medir el desempeño económico en los negocios de subsistencia es complicado, dado que rara vez tienen registros contables (Yescas, 2008), no tienen un control sobre sus ventas (Hernández Girón et al., 2007c), y operan con deficiencias en el proceso administrativo (Jaspe, 2009), lo que dificulta precisar la productividad del sector artesanal.

La innovación tiene un papel importante en el desempeño de los talleres artesanales, Hernández Girón et al. (2007b) analizan los factores internos y externos de la innovación empleados por los artesanos en ocho entidades de México. Los autores encontraron que el conocimiento 


\section{IIVESTIGAGIÓก Y CIECEIA DE LA UNIVERSIDAD AUTÓNOMA DE AGUASCALIERTES}

en el manejo del taller es más relevante que el conocimiento administrativo como factor explicativo de la innovación en este tipo de negocios. Además, agregan que con la finalidad de ser competitivos y de satisfacer los variados gustos de los consumidores, los artesanos establecen mecanismos para diferenciar su producto.

Domínguez Hernández et al. (2004), en un estudio realizado a 176 artesanos de Oaxaca, Jalisco y Guanajuato, muestran que la competitividad en este tipo de talleres - participación en el mercado, satisfacción con el negocio, índice de exportaciones, lealtad de los clientes y prestigio de la empresa- aumenta con la innovación mejoras y modificaciones a los productos, procesos, procedimientos y a las formas de comercialización-.

En el mismo sentido que los trabajos anteriores, Hernández Girón et al. (2007a) encontraron que la innovación administrativa —cambios, por ejemplo, a formas de organización, de distribución del trabajo y de productos- es uno de los factores que contribuyó al éxito - comparación del ingreso por ventas del año anterior y del actual- de 337 negocios artesanales en ocho estados de la República Mexicana.

Estudios específicos sobre este sector también demuestran que la innovación tiene un rol determinante en el desarrollo de estos negocios, por ejemplo: Lugo Morin et al. (2008) analizan la artesanía textil en el municipio de San Pablo Villa de Mitla, Oaxaca y encuentran que la demanda de mercado de los productos textiles de esta zona ha conllevado entre otros factores a la incorporación de innovaciones en los talleres artesanales tales como la integración de fibras sintéticas, diversificación, mejoras notables y mayor calidad en los productos artesanales, inclusión de instrumentos de trabajo modernos que incrementan la producción.

Ahora, ¿̇qué se debe entender por innovación? Para Alfred Marshall la innovación es una continua adaptación estructural a los cambios observados en el mercado de los factores de producción y de los productos elaborados, considera a la innovación como un criterio de la función empresarial. Otra perspectiva es la de Joseph Schumpeter (1935), quien hace énfasis en las nuevas empresas, los procesos tecnológicos y el incremento del liderazgo en los empresarios, define a la innovación como el establecimiento de una nueva función de producción.
Para la OECD (2006: 56) este término implica "la introducción de un nuevo, o significativamente mejorado, producto (bien o servicio), de un proceso, de un nuevo método de comercialización o de un nuevo método organizativo, en las prácticas internas de la empresa, la organización del lugar de trabajo - las relaciones exteriores". La misma OECD (2006) clasifica la innovación en cuatro formas:

1. De producto, cambios en las características de los bienes enteramente nuevos y mejoras significativas de los productos ya existentes.

2. De procesos, cambios en los métodos de manufactura, se caracteriza por modificaciones en las técnicas, materiales y/o sistemas informáticos.

3. De organización, implica la puesta en marcha de nuevos métodos o prácticas de organización.

4. De mercadotecnia, consiste en la adopción de nuevos métodos de comercialización.

Identificar la influencia de los factores de innovación en el desempeño económico de los talleres artesanales, representa soluciones nuevas que mejoren la efectividad, la eficacia y rentabilidad, lo que permite hacer frente a la incertidumbre económica y a la competencia en los mercados locales. Por tanto, el objetivo de este trabajo fue analizar la influencia de algunas variables de innovación sobre los ingresos por ventas en los talleres artesanales en la zona metropolitana de Zacatecas.

La hipótesis propuesta en el trabajo fue que cada una de las innovaciones de producto, proceso, organización y mercadotecnia influyen en el desenvolvimiento económico de talleres artesanales de la zona metropolitana de Zacatecas.

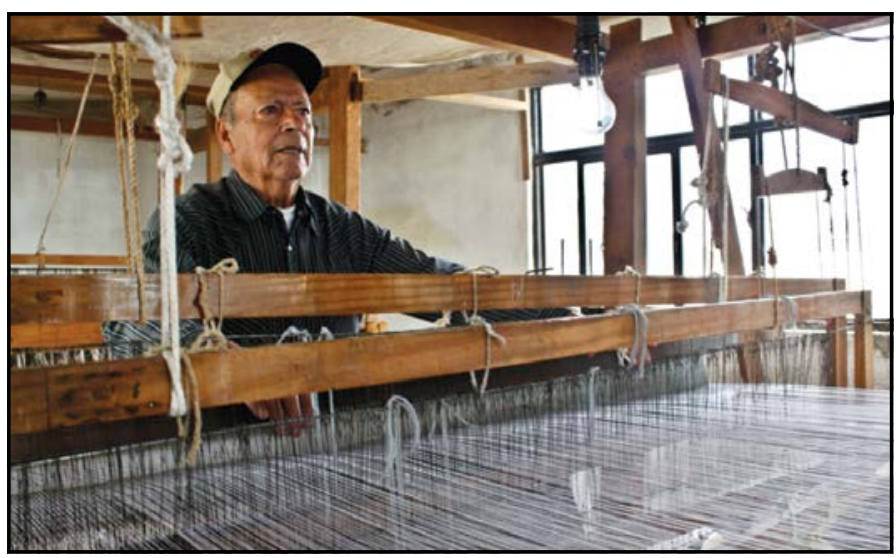

Figura 1. Artesano de la rama textil del municipio de Guadalupe trabajando en su telar de pedal. Fotografía tomada de la Subsecretaría de Desarrollo Artesanal. 


\section{IIVESTIGAGIÓn Y CUERCIA DE LA UกIVERSIDAD AUTÓOOOMA DE RGUASCHLIETTES}

\section{MATERIALES Y MÉTODOS}

La investigación se centró en los talleres artesanales de Zacatecas y su zona metropolitana, compuesta por los municipios de Guadalupe y Zacatecas, que se localizan en el centro del estado, en la demarcación señalada la principal fuente de ingresos son los servicios y el comercio. Aunque no existen estadísticas que reflejen la magnitud del sector artesanal, los negocios que se dedican a la producción de artesanías son importantes en la actividad económica de la región, ya que contribuyen con empleos para cientos de familias (Correa García y González Acolt, 2016).

La selección de la muestra se realizó con base en el padrón de artesanos del Instituto de Desarrollo Artesanal (IDEAZ; 2012), con un tamaño original de 167 talleres, los cuales se seleccionaron de acuerdo con la fórmula de determinación de la muestra con un nivel de confiabilidad de $95 \%$. El siguiente paso consistió en recolectar los datos, mediante un cuestionario aplicado por medio de entrevistas personales a los propietarios de cada negocio artesanal. La visita se realizó en el periodo comprendido desde el 1 hasta el 31 de octubre de 2014. El total de visitas fue de 167 negocios; sin embargo, no fue posible localizar a algunos artesanos debido a que fallecieron, diversos talleres cerraron, hubo errores en la base de datos; por tanto, el total de encuestas levantadas fue de 124.
Basados en los estudios de Salom Carrasco (2003); Hernández Girón et al. (2007a); Domínguez Hernández y Hernández Girón (2007); Hernández Girón et al. (2007b); Sánchez et al. (2010) y Correa (2014); se clasificó cada uno de los cuatro factores de la innovación —bajo el criterio de la OECD—que afectan el desempeño económico de los talleres artesanales de la zona metropolitana de Zacatecas (Tabla 1). La variable dependiente -desempeño económico- fue catalogada como los ingresos por venta en pesos que percibe el taller artesanal en un mes (INEGI, 2014).

Basados en la descripción de las variables de la Tabla 1, el modelo de regresión múltiple propuesto fue el siguiente:

$$
\begin{aligned}
& y=B_{0}+B_{1} X_{1}+\beta_{2} X_{2}+\beta_{3} X_{3}+\beta_{4} X_{4}+\beta_{5} X_{5}+\beta_{6} X_{6}+\beta_{7} X_{7}+\beta_{8} X_{8} \\
& +B_{9} X_{9}+\beta_{10} X_{10}+\beta_{11} X_{11}+\beta_{12} X_{12}+\beta_{13} X_{13}+\mu(1)
\end{aligned}
$$

Donde la variable y mide el ingreso en pesos que percibe el taller artesanal en un mes; ß1, B2... B13 son los parámetros a estimar, X1, X2:..X13 variables independientes que están definidas en la tabla, $\mu$ : es un término de error aleatorio. Las cuatro hipótesis propuestas a probar son las siguientes:

1) Para probar la hipótesis nula de que una vez controlada $\mathrm{X}_{5}, \mathrm{X}_{6}, \mathrm{X}_{7}, \mathrm{X}_{8}, \mathrm{X}_{9}, \mathrm{X}_{10}, \mathrm{X}_{11}, \mathrm{X}_{12} \mathrm{Y} \mathrm{X}_{13}$, los factores de innovación de producto no tienen efecto sobre el desempeño económico de los talleres artesanales

\begin{tabular}{|c|c|c|}
\hline Tipo de innovación & Preguntas & Escala \\
\hline De producto & 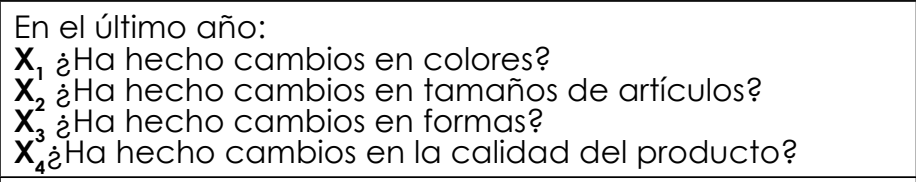 & $\begin{array}{l}\text { Variable dicotómica } \\
\qquad 1=\mathrm{Si}, 0=\mathrm{No}\end{array}$ \\
\hline De procesos & \begin{tabular}{|l}
$\mathbf{X}_{5} \dot{\mathrm{e}}$ Ha utilizado materiales mejorados? \\
$\mathbf{X}_{6} \dot{2} \mathrm{Ha}$ cambiado la forma de producir sus artículos?
\end{tabular} & $\begin{array}{l}\text { Variable dicotómica } \\
\qquad 1=\mathrm{Sí}, 0=\mathrm{No}\end{array}$ \\
\hline De organización & $\begin{array}{l}\mathbf{X}_{7} \text { i Ha realizado cambios a la organización del trabajo? } \\
\mathbf{X}_{8} \dot{H} \text { Ha buscado una mejor relación con sus clientes? } \\
\mathbf{X}_{9} \dot{2} \text { Ha buscado una mayor relación con sus } \\
\text { proveedores? } \\
\mathbf{X}_{10} \dot{2} \text { Ha buscado una mayor relación con sus compañeros } \\
\text { del taller? }\end{array}$ & $\begin{array}{l}\text { Variable dicotómica } \\
\qquad 1=\mathrm{Si}, 0=\mathrm{No}\end{array}$ \\
\hline De mercadotecnia & 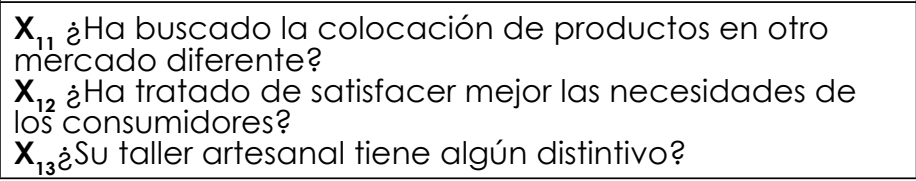 & $\begin{array}{l}\text { Variable dicotómica } \\
\qquad 1=\mathrm{Si}, 0=\mathrm{No}\end{array}$ \\
\hline
\end{tabular}

Tabla 1. Operacionalización de variables

La Tabla 1 describe los cuatro tipos de innovación (producto, proceso, organización y mercadotecnia), así como las preguntas realizadas, medidas en escala dicotómica. Elaboración propia. 
IIVESTIGACIÓn Y CIERCIA DE LA UחIVERSIDAD AUTÓNOTH

de la zona metropolitana de Zacatecas, se propone lo siguiente: $\mathrm{H}_{0}: B_{1}=0, B_{2}=0, B_{3}=0, B_{4}=0$; por tanto, la hipótesis alterna $\mathrm{H}_{1}: \mathrm{H}_{0}$ no es verdadera.

2) Por otra parte, la hipótesis nula de que la innovación en proceso no influye en el ingreso que perciben los artesanos de Zacatecas se plantea como $\mathrm{H}_{0}: B_{5}=0, B_{6}=0$, versus la hipótesis alterna $\mathrm{H}_{1}$ : $\mathrm{H}_{0}$ no es verdadera.

3 y 4) Similarmente, la hipótesis nula de que la innovación en organización y mercadotecnia, respectivamente, no ejercen influencia sobre el desenvolvimiento económico de los talleres artesanales de la zona metropolitana de Zacatecas se plantea para el caso de organización como $H_{0}: B_{7}=0, B_{8}=0, B_{9}=0, B_{10}=0 ; y$ para el caso de mercadotecnia como $H_{0}: B_{11}=0, B_{12}=0, B_{13}=0$, mientras que para ambos casos la hipótesis alterna implica que $\mathrm{H}_{1}$ : $\mathrm{H}_{0}$ no es verdadera.

\section{RESULTADOS}

Los estadísticos $F$ y chi-cuadrada se muestran en la Tabla 2, la primera distribución estima la relación de las varianzas de dos muestras tomadas independientemente de la misma población normalmente distribuida. La prueba chi-cuadrada compara la distribución observada de los datos con una distribución esperada de los mismos. Los grados de libertad son el número de valores que pueden ser asignados de forma arbitraria, antes de que el resto de las variables tomen un valor automáticamente. El valor de $p$ representa la probabilidad de aceptar la hipótesis de investigación.

Las distribuciones anteriores se emplean para probar la hipótesis 1, si consideramos el valor-p del estadístico $F$ existe cierta evidencia contra la hipótesis nula, específicamente, la $\mathrm{H}_{0}$ se rechaza a $10 \%$ pero no a $5 \%$.

Tabla 2. Resultado de la hipótesis innovación en producto

\begin{tabular}{|l|c|c|c|}
\hline $\begin{array}{c}\text { Prueba } \\
\text { estadística }\end{array}$ & Valor & $\begin{array}{c}\text { Grados de } \\
\text { libertad }\end{array}$ & Probabilidad \\
\hline F estadística & 3.70 & $(4,104)$ & 0.0074 \\
\hline Chi-cuadrada & 14.80 & 4 & 0.0051 \\
\hline
\end{tabular}

La Tabla 2 presenta los estadísticos $F$ y chi-cuadrada, los cuales se emplearon para probar la hipótesis 1 . Muestra los valores para cada prueba estadística, y el valor $p$, que es inferior al nivel crítico 0.05. Elaboración propia.
La Tabla 3 ofrece el listado de la prueba estadística $F$ y chi-cuadrada, ambos se emplean para probar la hipótesis 2, el valor $p$ para ambos estadísticos es grande, por lo cual es muy débil la evidencia contra la hipótesis nula; este resultado sugiere que la innovación en proceso, contrario a lo que se esperaba, no ejerce influencia significativa en el desempeño económico de los negocios artesanales.

Tabla 3. Resultado hipótesis innovación en proceso

\begin{tabular}{|l|c|c|c|}
\hline \multicolumn{1}{|c|}{$\begin{array}{c}\text { Prueba } \\
\text { estadística }\end{array}$} & Valor & $\begin{array}{c}\text { Grados de } \\
\text { libertad }\end{array}$ & Probabilidad \\
\hline F estadística & 0.89 & $(2,104)$ & 0.4106 \\
\hline Chi-cuadrada & 1.80 & 2 & 0.4074 \\
\hline
\end{tabular}

La Tabla 3 presenta el resultado de la regresión múltiple, aquí podemos apreciar las pruebas estadísticas $F$ y chi-cuadrada, donde se contrasta la hipótesis nula, p >0.05; por tanto, se acepta la hipótesis nula. Elaboración propia.

Para probar la hipótesis 3, se obtuvieron los estadísticos de la variable innovación de organización que aparecen en la Tabla 4, se puede apreciar que los valores $p$ de los dos estadísticos son inferiores al nivel de $10 \%$, lo cual muestra evidencia fuerte contra la $\mathrm{H}_{0}$.

Tabla 4. Resultado de la hipótesis innovación en la organización

\begin{tabular}{|l|c|c|c|}
\hline $\begin{array}{c}\text { Prueba } \\
\text { estadística }\end{array}$ & Valor & $\begin{array}{c}\text { Grados de } \\
\text { libertad }\end{array}$ & Probabilidad \\
\hline F estadística & 2.32 & $(4,104)$ & 0.0613 \\
\hline Chi-cuadrada & 9.30 & 4 & 0.0540 \\
\hline
\end{tabular}

La Tabla 4 presenta los estadísticos $F$ y chi-cuadrada, los cuales se emplearon para probar la hipótesis 3 , muestra los valores para cada prueba estadística, y la probabilidad que es inferior al nivel 0.10. Elaboración propia.

Los estadísticos relacionados con la innovación en mercadotecnia aparecen en la Tabla 5 y se emplearon para probar la hipótesis 4; los valores $p$ para los dos estadísticos son inferiores al nivel crítico de $1 \%$, este resultado permite rechazar la hipótesis nula, por lo que la innovación de mercadotecnia sí tiene influencia en los ingresos de los artesanos de la zona metropolitana de Zacatecas.

\section{DISCUSIÓN}

La innovación en producto ejerce influencia significativa en los ingresos de los artesanos del área metropolitana de Zacatecas, este hallazgo es congruente con los de Domínguez et al. (2004), 


\section{IIVESTIGAGIÓn Y CUERGIA DE LA UNIVERSIDAD AUTÓnOMH DE RGUASCALIERTES}

Tabla 5. Resultado hipótesis innovación en mercadotecnia

\begin{tabular}{|l|c|c|c|}
\hline $\begin{array}{c}\text { Prueba } \\
\text { estadística }\end{array}$ & Valor & $\begin{array}{c}\text { Grados de } \\
\text { libertad }\end{array}$ & Probabilidad \\
\hline F estadística & 4.13 & $(3,104)$ & 0.0082 \\
\hline Chi-cuadrada & 12.39 & 3 & 0.0061 \\
\hline
\end{tabular}

En la Tabla 5 se pueden apreciar las pruebas estadísticas $F$ y chi-cuadrada donde se contrasta la hipótesis nula, con un valor $p<0.01$; por tanto, no se rechaza la hipótesis nula. Elaboración propia.

quienes sostienen que la innovación es una variable relevante para que el artesano se dedique a elaborar productos novedosos, diferentes y atractivos que le generarán un mayor volumen de ventas $y$, por lo mismo, un incremento en sus utilidades.

Referente a la innovación en proceso, esta variable no ejerce influencia significativa en el desempeño económico de los negocios artesanales. Esto puede ser explicado debido a que las piezas de artesanía son producidas con apego a procesos tradicionales heredados por generaciones; por tanto, resulta difícil cambiar la forma de producir sus artículos y rara vez utilizan materiales mejorados.

La innovación de organización sí ejerce influencia en los ingresos de los artesanos, este hallazgo es similar al de Hernández Girón et al. (2007b), quienes en un primer momento del análisis - en la etapa de definición de la estructura conceptual de las diferentes innovaciones-, encuentran que elementos como la mayor relación con los clientes y con proveedores tienen una carga factorial significativa en la innovación organizacional de los talleres artesanales.

La variable de innovación en mercadotecnia, al igual que la anterior, sí ejerce influencia sobre el desempeño económico de los talleres artesanales. Ello concuerda con lo encontrado en el estudio de Jiménez Castañeda et al. (2009), quienes hallaron correlaciones significativas entre las estrategias de mercadotecnia y competitividad de 337 talleres artesanales ubicados en Michoacán, Estado de México, Guanajuato, Puebla, Jalisco, Guerrero, Oaxaca y Veracruz.

CONCLUSIONES

La innovación es crucial en el desempeño de los talleres artesanales, la capacidad de adaptación al entorno y la resolución de problemas. Al realizar innovación en producto los artesanos ofrecen artesanías diferentes, mayor facilidad de venta, mejor manejo de precios, lo que repercute en mayores utilidades.

La innovación en proceso es la menos utilizada, porque trabajan con procesos artesanales ancestrales, transmitidos de generación en generación. Los talleres artesanales están compuestos en su mayoría por miembros de la misma familia, razón por la cual realizan innovación en la organización, esto debido a que el artesano rara vez puede producir y vender a la vez, y tiene que dividir las actividades de producción o de comercialización con sus hijos. La adopción de nuevos métodos de comercialización en el taller artesanal a través de la innovación de mercadotecnia permite al negocio incrementar su presencia en el mercado, atraer mayor número de clientes, ello representa un incremento en ventas; por tanto, generan una mejora en el desempeño económico, pues los artesanos prefieren innovaciones que exijan pocos recursos financieros.

La identificación de la influencia de los factores de innovación en el desempeño económico de las empresas familiares artesanales representa alternativas que les permiten ser más competitivos y rentables, abandonar el nivel de subsistencia y hacer frente a la incertidumbre económica y la competencia en los mercados locales. La limitación primordial es el tamaño de la muestra, ya que es recomendable realizar estudios con mayor número de artesanos y, de preferencia, de otras ramas. 
- BURCIAgA, J. Zacatecas, memoria sobre el arte popular. México: CONACULTA, 2010.

- CORREA, L. La innovación en producto en los talleres artesanales de Zacatecas y de la República Mexicana. Congreso Internacional de Investigación e Innovación 2014. Cortázar, Guanajuato, mayo 2014.

- correa garcía, L. A. y GOnzÁlez acolt, R. el sector artesanal en México y el combate contra la pobreza. Transitare Revista de Turismo, Economía y Negocios, 2(2): 233250, 2016.

- DOMíngueZ hERNÁNDEZ, M. L. y HERNÁNDEZ GIRÓN, J. P. Impacto, a dos años, de los programas del FONART en los indicadores de pobreza, producción y comercialización artesanal. Informe Técnico Final. Tomo 1. México: Instituto Politécnico Nacional, 2007.

- DOMÍNGUEZ HERNÁNDEZ, M. L. et al. Competitividad y ambiente en sectores fragmentados. El caso de la artesanía en México. Cuadernos de administración, 17(27): 127-158, 2004.

- HERNÁNDEZ GIRÓN, J. et al. Factores de éxito en los negocios de artesanía en México. Estudios gerenciales, 23(104): 77-99, 2007a.

- HERNÁNDEZ GIRÓN, J. P. et al. Factores de innovación en negocios de artesanía de México. Gestión y Política Pública, 16(2): 353-379, 2007b.

- HERNÁNDEZ GIRÓN, J. P. et al. Orientación al mercado y desempeño en artesanías. Naturaleza y Desarrollo, 5(1): 2740, 2007c.

- IDEAZ (INSTITUTO DE DESARROLLO ARTESANAL DE ZACATECAS). Padrón de artesanos. Gobierno del Estado de Zacatecas, septiembre 2012.

- JASPE, C. Diagnóstico regional de artesanías. Zacatecas: Instituto de Desarrollo Artesanal del Estado de Zacatecas, octubre 2009.
- JIMÉNEZ CASTAÑEDA, J. C. et al. Estrategias y competitividad de los negocios de artesanía en México. Pensamiento y Gestión, 26, 165-190, 2009.

- LUGO MORIN, D. R. et al. Etnocompetitividad del sistema artesanal textil Mitla, el papel del territorio y la innovación. Economía, Sociedad y Territorio, 8(28): 981-1006, 2008.

- ocde (ORGANIZACIÓN DE COOPERACIÓN Y DESARROLLO ECONÓMICOS)-EUROSTAT (OFICINA DE ESTADÍSTICAS DE LAS COMUNIDADES EUROPEAS). Manual de Oslo, Guía para la recogida e interpretación de datos sobre innovación. 3 ed. OCDE-EUROSTAT, 2006.

- SALOM CARRASCO, J. Innovación y actores locales en los nuevos espacios económicos: Un estado de la cuestión. Boletín de la Asociación de Geógrafos Españoles, 36, 7-30, 2003.

- SÁNCHEZ, P. S. et al. Género y comportamiento ambiental de los negocios de artesanías de barro. Gestión y Política Pública, XIX(1): 79-110, 2010.

- SCHUMPETER, A. Análisis del cambio económico. The Review of Economics Statics, XVII(4): 2-10, 1935.

- TOledo LÓPEZ, A. et al. Defining success in subsistence businesses. Journal of Business Research, 65(12): 1658-1664, 2012.

- YeSCAS, M. La cultura organizacional y el desempeño de los negocios de artesanía. México: Instituto Politécnico Nacional, 2008.

\section{De páginas electrónicas}

- INEGI (INSTITUTO NACIONAL DE ESTADístiCA y GEOGRAFÍA). Encuesta en hogares/micronegocios 2012. Recuperado de http://www.beta.inegi.org.mx/proyectos/enchogares/ modulos/enamin/2012/ 